\title{
Capsule Commentary on Silverman et al., The Relationship Between Food Insecurity and Depression, Diabetes Distress and Medication Adherence Among Low-Income Patients with Poorly Controlled Diabetes
}

\author{
Joanna Buscemi, PhD \\ Institute for Health Research and Policy, University of Illinois at Chicago, Chicago, IL, USA.
}

J Gen Intern Med 30(10): 1533

DOI: $10.1007 / \mathrm{s} 11606-015-3407-2$

(c) Society of General Internal Medicine 2015

$\mathrm{T}$ he most proximal predictor of the behavioral management of any chronic disease is engaging in health-promoting behaviors. In the case of diabetes control, adherence is everything. Maintaining a healthy diet, consistent blood sugar monitoring, and medication compliance are key components to improving diabetes outcomes. But what happens when individuals have multiple barriers to adherence and do not live in an environment that supports these health behaviors?

The Social Ecological Model ${ }^{1}$ depicts the importance of conceptualizing health behaviors beyond the individual, and also considering interpersonal, community, and policy-level variables. Social determinants of health are an important consideration when problem solving around barriers to disease management. Individuals with food insecurity have limited access to affordable, healthful foods. ${ }^{2}$ Food insecurity is not only related to poor nutrition, but also depression, low compliance to medication, higher self-reported disease-related distress, and poorer health outcomes overall.

Silverman and colleagues ${ }^{3}$ examine variables associated with food insecurity as they relate to diabetes management among low-income adults with diabetes. Their findings suggest that assessing for food insecurity among diabetic patients in primary care might have clinical implications to help improve outcomes (e.g., referral psychologist to treat depression or problem solve around medication adherence).
Such screening may require changes in institutional policies in our hospitals and clinics, and would require working on interprofessional teams to improve quality of care. But even beyond that, the paper speaks to a larger issue. How can we, as researchers and clinicians, advocate for change in our communities to increase access to healthful foods? How can we advocate for change at city or state levels to inform policies that might help improve the overall health of our patients? Just as patient barriers to adherence to treatment go beyond the individual level and outside of the office doors, impacting these barriers may also require intervention outside of our doors and into our communities.

Conflict of Interest: The author has no conflicts of interest with this article.

Corresponding Author: Joanna Buscemi, PhD; Institute for Health Research and Policy, University of Illinois at Chicago, Chicago, IL, USA (e-mail:jbuscemi@uic.edu).

\section{REFERENCES}

1. McLeroy KR, Steckler A, Bibeau D, Eds. The social ecology of health promotion interventions. Health Educ Q. 1988; 15(4):351-377. Retrieved May 6, 2015, from http://tamhsc.academia.edu/KennethMcLeroy/Papers/ 81901/An_Ecological_Perspective_on_Health_Promotion_Programs.

2. Anderson SA, ed. Core Indicators of nutritional state for difficult-to-sample populations. J Nutr. 1990; 120(11S): 1557-1600. Report by the Life Sciences Research Office, Federation of American Societies for Experimental Biology, for the American Institute of Nutrition.

3. Silverman J, Krieger J, Kiefer M, Hebert P, Robinson J, Nelson K. The relationship between food insecurity and depression, diabetes distress and medication adherence among low-income patients with poorly-controlled diabetes. J Gen Intern Med. 2015. doi:10.1007/s11606-015-3351-1. 